\title{
A 9-year-old girl presented with jaundice, abdominal distension, hema- temesis, melena and pleural effusion
}

\author{
Parisa Marjan, Md. Rukunuzzaman, A. S. M. Bazlul Karim, Mohuya Mondal and Hazera Akter
}

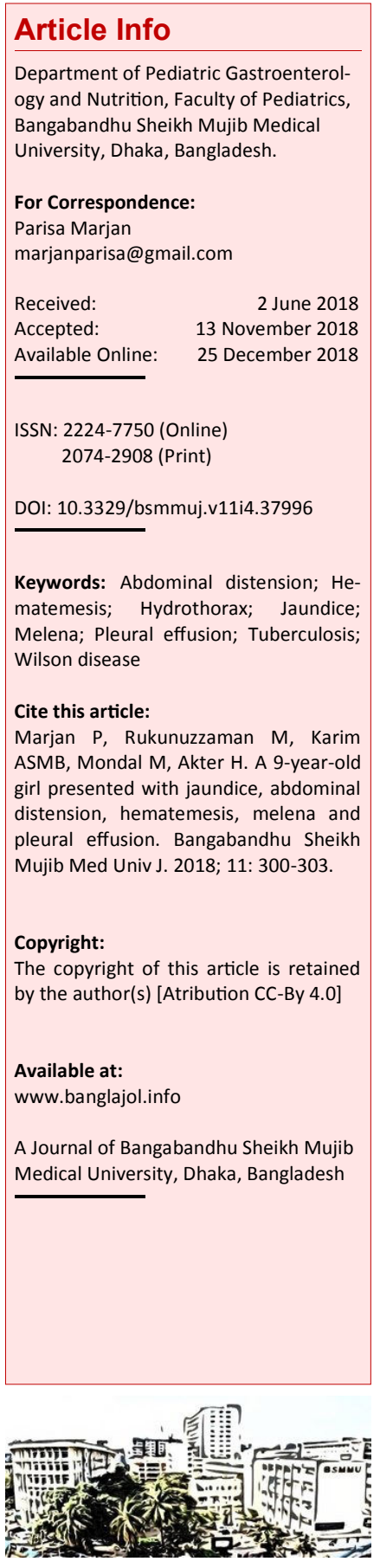

\section{Presentation of Case}

Dr. Parisa Marjan (MD Resident): A 9 year old girl of non-consanguineous parents presented at the outpatient department with the history of jaundice and abdominal distension for 20 days and respiratory distress for 7 days. She also complained of bloody vomiting without any melena. The mother gave the history of abdominal pain for 2 days which was diffuse in nature. Her menarche had not started yet. Her younger sister is healthy. She had no history of fever, constipation, family history of liver disease, sib death, contact with known tuberculosis patient, blood transfusion or parenteral medication.

On examination, she was afebrile, moderately pale, dyspnea and leukonychia. Bilateral pedal edema was present. Per abdominal examination revealed splenomegaly $(4 \mathrm{~cm})$ and ascites. On examination of the respiratory system revealed breath sound was diminished in the left side from $2^{\text {nd }}$ intercostal space downward. Other systemic examinations were normal.

Initial laboratory investigations showed hemoglobin $7.6 \mathrm{~g} / \mathrm{dL}$, total white blood cell count $5000 / \mathrm{mm}^{3}$ (neutrophil- $47 \%$, lymphocyte - $46 \%$ ) and platelet $115,000 / \mathrm{mm}^{3}$ (Table I). The liver function test showed alanine aminotranferase $81 \mathrm{U} / \mathrm{L}$, albumin $16 \mathrm{~g} / \mathrm{l}$, prothrombin time $41 \mathrm{sec}$, international normalized ratio 3.4. Serum electrolyte was hypokalemic $\left(\mathrm{K}^{+} 3.0\right.$ $\mathrm{mmol} / \mathrm{L})$. The HBsAg and anti-HCV were negative. The slit lamp examination of the eye showed bilateral Kayser-Fleisher ring. 24 hour urinary copper was $319 \mu \mathrm{g} /$ day and the serum cerulo-plasmin was $10 \mathrm{mg} / \mathrm{dL}$. The upper gastro-intestinal endoscopy was done which revealed Grade 1 varices. The chest X-ray showed left-sided pleural effusion (Figure 1). Other investigations were done to exclude the common causes of unilateral pleural effusion. Tuberculin test was negative. Echocardiography was normal. The serum lactate dehydrogenase level was normal. The antinuclear antibodies test was negative. The findings of the pleural fluid study were: protein $6 \mathrm{~g} / \mathrm{L}$ (transudative), glucose $6.3 \mathrm{mmol} / \mathrm{L}$. cell count
$20 / \mathrm{mm}^{3}$ with $90 \%$ lympho-cyte, no malignant cell was present. The pleural fluid lactate dehydrogenase and lipase were normal, The pleural fluid adenosine deaminase was negative.

Based on the history, clinical examination and investigations, the provisional diagnosis was made.

\section{Provisional Diagnosis}

Wilson disease with hepatic hydrothorax

\section{Differential Diagnosis}

\section{Tuberculous pleural effusion}

Tuberculosis is the important cause of pleural effusion. 1 Tuberculous pleural effusion is considered as extrapulmonary tuberculosis where tuberculosis is endemic.? It is synonymous with tuberculous pleurisy. The incidence of pleural involvement is high (up to 30\%) in many areas. Tuberculous pleural effusion usually presents with fever, cough and chest pain. The pleural fluid is exudative in nature and has lymphocyte predominance. The gold standard for the diagnosis of tuberculous pleural effusion is the detection of Mycobacterium tuberculosis in the pleural fluid or pleural biopsy specimen or the presence of caseating granulomas in the pleura along with bacilli. Adenosine deaminase in the pleural fluid has been documented to be a useful test for the diagnosis. It the area with high tuberculosis prevalence, the easy way to establish the diagnosis of tuberculous pleural effusion in a patient with a lymphocytic pleural effusion is to level of adenosine deaminase above $40 \mathrm{U} / \mathrm{L} . \underline{3}$

Dr. Marjan: After evaluating the patient's presenting features, physical findings and the laboratory tests, the pleural fluid study was done. The pleural fluid study revealed, protein $6 \mathrm{gm} / \mathrm{L}$ (transudative), glucose $6.3 \mathrm{mmol} / \mathrm{L}$. Cytology showed the cell count $20 / \mathrm{mm}^{3}$ with $90 \%$ lymphocyte with no malignant cell. The pleural fluid lactate dehydrogenase and lipase 
Table I

\begin{tabular}{|lrr|}
\multicolumn{3}{c}{ Laboratory investigations } \\
Parameter & Patient & References \\
\hline Hemoglobin (g/dL) & 7.6 & $13.5 \pm 1.3$ \\
Total white blood cell count (×109/L) & 5 & $7.0 \pm 3$ \\
Differential count & & \\
$\quad$ Neutrophil (\%) & 47 & $40-80 \%$ \\
$\quad$ Lymphocyte (\%) & 46 & $20-40 \%$ \\
$\quad$ Eosinophil (\%) & 3 & $1-06 \%$ \\
$\quad$ Monocyte (\%) & 4 & $2-10 \%$ \\
Platelet count (x10 $/ \mathrm{L})$ & 115 & $150-400$ \\
Serum alanine aminotransferase (U/L) & 81 & $30-65$ \\
Serum albumin (g/L) & 16 & \\
Prothrombin time (sec) & 41 & \\
International normalized ratio & 3.4 & $3.5-5.2$ \\
\hline Serum potassium (mmol/L) & 3.0 & \\
\hline
\end{tabular}

levels were normal. The pleural fluid adenosine deaminase level was below the level for the diagnosis of tuberculosis.

\section{Dr. Marjan's Diagnosis}

Hepatic hydrothorax

\section{Discussion}

Dr. Subarna Das: Why it is not a case of lymphoma?

Dr. Mohuya Mondal: The pleural effusion is a common presenting feature of lymphoma. The Tcell lymphoma mainly causes ascites and pleural effusion. In the case of Hodgkin's disease, the patho
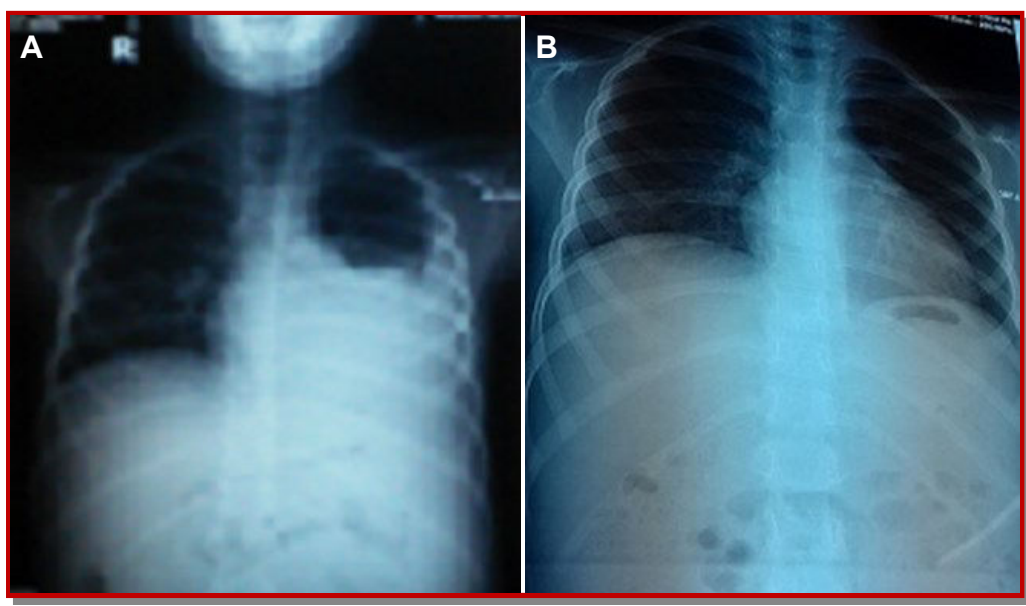

Figure 1: Chest X-ray showing the right-sided hydrothorax (A) and after treatment (B) -genesis of pleural effusion is reduced lymphatic drainage due to obstruction of the thoracic duct. But in non-Hodgkin's lymphoma, the mechanism is the direct infiltration of pleura by the tumor mass. The diagnosis is confirmed by the pleural fluid study for the cytology and immunophenotyping. $\underline{4}$ As this patient had no history of fever, drenching night sweating, weight loss or lymphadenopathy. The serum lactate dehydrogenase was normal and the pleural fluid study was non-hemorrhagic and negative for the malignant cell.

Dr. Archana Shrestha Yadav: Could it be a case of pancreatitis?

Dr. Mondal: The pleural effusion may occur as a complication of acute or chronic pancreatitis. In acute pancreatitis, there may be the left-sided and small amount of pleural fluid collection. The mechanism is the reactive inflammation of the pleura due to pancreatitis. In the course of chronic pancreatitis, the pleural effusion is related to the presence of pancreaticopleural fistula. Here, the pleural effusion is large in amount, recurrent, single -sided or bilateral. But this patient had no characteristic abdominal pain with vomiting or fever or any feature of exocrine or endocrine insufficiency which clinically exclude pancreatitis. $\underline{5}$

Dr. Kamrun Nahar: Why is it not spontaneous bacterial pleuritis?

Dr. Mondal: The spontaneous bacterial pleuritis is a major complication of the hepatic hydrothorax. In spontaneous bacterial pleuritis, the patient usually presents with fever, a feature of pleuritic chest pain with or without the feature of encephalopathy. The mechanism is due to the direct bacterial spread from the peritoneal cavity transiently. This patient has no suggestive clinical feature of spontaneous bacterial pleuritis. Moreover, polymorphonuclear leukocyte count was low $\left(20 / \mathrm{mm}^{3}\right)$ in the pleural fluid along with high serum ascites albumin gradient.

Dr. Md. Benzamin: What management did you give to the patient?

Dr. Marjan: We managed the child with salt restricted diet, injection cefotaxime, injection flucloxacillin, infusion of human albumin, oral furosemide, and transfusion of packed red blood cell. After confirmation of the diagnosis of Wilson disease, we added the penicillamine, zinc, pyruvate along with copper restricted diet.

Dr. Luthfunnahar: In patient of hepatic hydrothorax on which side of pleural effusion is common and why?

Dr. Hazera Akter: In case of hepatic hydrothorax, the right-sided pleural effusion is common 6 , as because the right-sided diaphragm is thinner and small fenestrations are present in the right side. $?$ 
Dr. Sayma Munmun: Is there any role of therapeutic thoracocentesis in case of hepatic hydrothorax?

Dr. Mondal: Yes. Thoracocentesis has both the diagnostic and therapeutic roles. In case of uncomplicated hepatic hydrothorax: cell count- $<1000 / \mathrm{mm}^{3}$, polymorphonuclear leukocyte count- $<250 / \mathrm{mm}^{3}$, protein- $<2.5 \mathrm{~g} / \mathrm{dL}$, pleural fluid/serum total protein ratio $<0.5$, pleural fluid : serum lactate dehydrogenase ratio- $>0.6$, pleural fluid/serum albumin gradient- $>1.1, \mathrm{pH}>7.4$. As this patient's pleural effusion was not massive clinically and radiologically, we only did the diagnostic thoracocentesis.

Dr. Saidul Islam Sumon: Why human albumin infusion was given?

Dr. Hazera Akter: The cause of pleural effusion of this patient is the chronic liver disease, where albumin production is low from the hepatocyte. That results in the reduced colloidal osmotic pressure in the blood and causing third spacing of fluid. So, the human albumin infusion was given to raise the colloidal osmotic pressure and reduce the ascites as well as pleural effusion. $\underline{8}$

Dr. Subir Ananda Biswas: Is there any need for surgical treatment?

Dr. Marjan: As the patient responded to medical management and did not need repetitive thoracocentesis, we did not think of surgical treatment.

Dr. Rana Kumar Biswas: What was your management for the esophageal varices?

Dr. Mondal: The patient has Grade 1 esophageal varices. So, we gave the tablet propranolol for primary prophylaxis of gastrointestinal bleeding. 9 Advice to take prompt treatment for any fever, cough or constipation and will do follow up endoscopy after 6 months.

Dr. Md. Rukunuzzaman: Hepatic hydrothorax is an uncommon presentation of a patient with portal hypertension due to liver cirrhosis. Hepatic hydrothorax is defined as the pleural effusion in a patient of liver cirrhosis, transudative in nature without having pulmonary or cardiac etiology with or without having ascites. The pleural fluid is usually $>500 \mathrm{~mL} . \underline{10}$ The estimated prevalence of this debilitating complication in patients with liver cirrhosis is $4 \%$ to $10 \%$.11 Pleural effusion is commonly in the right side $(85 \%)$, then left-sided $(13 \%)$ and bilateral $(2 \%) \underline{12}$

The proposed explanation of the development of hepatic hydrothorax is the presence of some microscopic defect in the diaphragm causing small peritoneal herniation and formation of pleuro-peritoneal bleb. During raised intra-abdominal pressure as in coughing, vomiting and ascites, the rupture of bleb occurs which causes leakage of ascitic fluid in the pleural spaces. $\underline{13}$ The negative intrathoracic pressure during inspiration helps to accumulate the ascitic fluid in the pleural cavity. Other explanations are the reduced colloid osmotic pressure due to hypoalbuminemia, increased azygos venous pressure and leakage of plasma into the pleural cavity, transmigration of peritoneal fluid into the pleural cavity through lymphatic channel. $\underline{14}$

The microscopic examination shows the discontinuity of collagen bundle in the tendinous part of the diaphragm where pleura-peritneal bleb occurs. Typically, the defect is $<1 \mathrm{~cm}$ and commonly occurs on the right side. This right $\square$ sided predominance is due to the thicker left side of the diaphragm and more muscular than the right side. $\underline{\underline{15}}$

The clinical manifestations are non-specific and related to ascites and features of portal hypertension due to decompensated liver disease. The respiratory symptoms in a patient with hepatic hydrothorax varies, mainly depends on the volume of effusion, rapidity of the effusion accumulation in the pleural space and the presence of cardiopulmonary disease. The patient may be asymptomatic and the pleural effusion may be the incidental finding on chest imaging performed for other reason. The patient may have pulmonary symptoms of shortness of breath, cough, hypoxemia or respiratory failure associated with large pleural effusions. $\underline{16}$

To diagnose the hepatic hydrothorax, cardiopulmonary dysfunction should be excluded first. It is required to establish the presence of transudative pleural fluid and the demonstration of diaphragmatic defect when possible. The investigations are done to establish the transudative pleural effusion and exclude infection, malignant or other causes. 17

Treatment of hepatic hydrothorax includes reducing the portal hypertension which is responsible for ascites formation, preventing the escape of fluid via diaphragmatic defects and draining of pleural fluid.18 In case of the majority of patients present with the end-stage liver disease, the treatment option is the orthotopic liver transplantation. The medical management includes salt-restricted diet, diuretics, and albumin infusion (if indicated). Repeated thoracocentesis may be required in some patients. 19 In patients with refractory hydrothorax, recommended therapies include the therapeutic thoracentesis, transjugular intrahepatic portal systemic shunt and pleurodesis.20 Chest drain insertion with subsequent chemical pleurodesis, is considered as a possible therapeutic strategy. Successful pleurodesis through a chest drain has been done in some patients with hepatic hydrothorax. $\underline{21}$

Spontaneous infection within the pleural fluid is known as spontaneous bacterial empyema. It is defined as pleural fluid with a polymorphonuclear cell count $>500$ cells $/ \mathrm{mm}^{3}$ or positive culture with polymorphonuclear cell count $>250$ cells $/ \mathrm{mm}^{3}$ with 
the exclusion of a parapneumonic effusion. 22 Hepatic hydrothorax may be complicated by spontaneous bacterial empyema, which has a poor prognosis with a mortality rate of up to $20 \% . \underline{23}$

This type of infection must be considered in any patient with hydrothorax who develops fever, pleuritic pain, encephalopathy, or unexplained deterioration in clinical status. A high index of suspicion is essential for its diagnosis, spontaneous bacterial empyema can be present in as many as $13 \%$ of patients with hepatic hydrothorax on admission with an associated mortality as high as $20 \%$ during treatment. It might occur as a result of a direct bacterial spread from the peritoneal cavity. Patients with advanced liver disease, low pleural fluid protein, or spontaneous bacterial peritonitis are at risk for spontaneous bacterial pleuritis. A diagnostic thoracentesis with subsequent culture of pleural fluid should be performed in cirrhotic patients with hydrothorax when infection is suspected. $\underline{24}$ Long-term survival of spontaneous bacterial empyema is poor, and thus liver transplant should be considered. As with spontaneous bacterial peritonitis, prophylaxis should be initiated in survivors of spontaneous bacterial empyema. $2 \underline{2}$

\section{Final Diagnosis}

Hepatic hydrothorax due to Wilson disease.

\section{References}

1. Vorster MJ, Allwood BW, Diacon AH, Koegelenberg CFN. Tubercular pleural effusion: Advances and controversies. J Thorac Dis. 2015; 7: 981-91.

2. Antonangelo L, Vargas FS, Puka J, Seiscento M, MM, Teixeira LR, Terra RM, Sales RKB. Pleural tuberculosis: Is radiological evidence of pulmonary -associated disease related to the exacerbation of the inflammatory response? Clinics 2012: 67: 125963.

3. Zhai K, Lu Y, Shi HZ. Tuberculous pleural effusion. J Thorac Dis. 2016; 8: 486-94.

4. Das DK. Serous effusions in malignant lymphoma; a review. Diagn Cytopathol. 2006; 34: 335-47.

5. Kamath S, Samanta RP, Rao BS. Pancreatic pleural effusion: A diagnosis, not to be missed! Arch Med Health Sci. 2016; 4: 216-21.

6. Roussos A, Philippou N, Mantzaris JG. Hepatic Hydrothorax: Pathophysiology diagnosis and management. J Gastroenterol Hepatol. 2007; 22: 1388-93.

7. Begum N, Karim ASM, Majumder W. Hepatic hydrothorax: An unusual presentation of Wilsons disease: A case report with review of relevent literature. Bangladesh J Child Health. 2012; 36: 10104 .

8. Bernardi M, Ricci CS, Zaccherini G. Role of human albumin in the management of complications of liver cirrhosis. J Clin Exper Hepatol. 2014; 4: 302-11.

9. Chen YI, Ghali P. Prevention and management of gastroesophageal varices in cirrhosis. Int J Hepatol. 2012; 2012.

10. Krishnamoorthy TL, Taneja M, Chang PE. Symptomatic hepatic hydrothorax successfully treated with transjugular intrahepatic portosystemic shunt (TIPS): Role of titration of portosystemic gradient reduction to avoid post-TIPS encephalopathy. Clin Case Rep. 2014; 2: 93-97.

11. Lazaridis KN, Frank JW, Krwka MJ, Kamath PS. Hepatic hydrothorax: Pathogenesis, diagnosis, and management. Am J Med. 1999: 3; 262-67.

12. Abbasi A, Bhutto AR, Alam MT, Aurangzaib M, Masroor M. Frequency of hepatic hydrothorax and its association with child pugh class in liver cirrhosis patients. J Coll Phys Surg Pakistan. 2016; 26: 566-69.

13. Doraiswamy V, Riar S, Shrestha P, Pi J, Alsumrain M, Bennet-Venner A, Kam J, Klukowicz A, Miller R. Hepatic hydrothorax without any evidence of ascites. Sci World J. 2011; 11: 587-91.

14. Siddappa PK, Kar P. Hepatic hydrothorax. Trop Gastroenterol. 2009; 30: 135-41.

15. Krok KL. Hepatic hydrothorax: Current concepts. Clin Liver Dis. 2014; 4: 35-37.

16. Singh A, Bajwa A, Shujaat A. Evidence-based review of the management of hepatic hydrothorax. Respiration 2013; 86: 155-73.

17. Lv Y, Han G, Fan D. Hepatic hydrothorax. Annals Hepatol. 2018; 17: 33-46.

18. Kashinathan G, Pillai N. A rare presentation of hepatic hydrotorax in a patient with alcoholinduced liver cirrhosis. Am J Med Case Rep. 2017; 5: 104-06.

19. Cardenas A, Kelleher T, Chopra S. Hepatic hydrothorax. Aliment Pharmacol Ther. 2004; 20: 271- 279.

20. Orman ES, Lok AFS. Outcome of patient with chest tube insertion for hepatic hydrothorax. Hepatol Int. 2009; 3: 582-86

21. Sukcharoen K, Dixon S, Mangat K, Stanton A. Case report: Hepatic hydrothorax in the absence of ascites. BMJ Case Rep. 2013; 2013.

22. Naglaa A, Allam H. Spontaneous bacterial empyema in liver cirrhosis: An underdiagnosed plural complication. Saudi J Gastroenterol. 2008; 14: 43-45.

23. Garcia NJr, Mithas AA. Hepatic hydrothorax: Pathophysiology, diagnosis and management. Clin Gastroenterol 2004; 38: 52-58.

24. Mansour AE, El-Rahman AA, Besheer T. Prevalence and risk factors for spontaneous bacterial pleuris in cirrhotic patients with hydrothorax. Egyptian J Chest Dis Tuberc. 2013; 62: 435-38.

25. Chertoff J, Nathoo S. Decompensated liver cirrhosis presenting as a spontaneous left sided bacterial empyema. ACG Case Rep J. 2016; 3: 124-26. 